\title{
Participación de los stakeholders en la gobernanza corporativa: fundamentación ontológica y propuesta metodológica
}

\section{Participation of stakeholders in corporate governance: foundation ontological and methodological proposal}

Recibido: 18 de noviembre de 2009 | Revisado: 19 de febrero de 2010 | Aceptado: 14 de diciembre de 2011

\author{
Leire SAN-Jose Ruiz De Aguirre* \\ University of Huddersfield, Reino Unido \\ Universidad del País Vasco, España \\ José LUIS RETOLAZA** \\ AURKILAN Business Ethics Research Institute \\ Instituto de Economía Aplicada a la Empresa, \\ UPV/EHU, España
}

SICI: 1697-9267(201206)11:2<619:PDSEGC>2.0.TX;2-E

Para citar este artículo. San-Jose, L. \& Retolaza, J. L. (2012). Participación de los stakeholders en la gobernanza corporativa: fundamentación ontológica y propuesta metodológica. Universitas Psychologica, 11(2), 619-628.

Profesora agregada de la Universidad del País Vasco (España) y Visiting Research Fellow de la University of Huddersfield (UK).E-mail: leire.sanjose@ ehu.es. ResearcherID: San-Jose, L. D9125-2011.

** Presidente del Grupo INTEGRA-Equalia grupo Empresarial y de Consultoría. Director del Instituto AURKILAN y Senior Research del Instituto de Economía Aplicada a la Empresa de la Universidad del País Vasco. E-mail: joseluis.retolaza@ehu.es

\section{RESUMEN}

La participación en la empresa es un tema de larga trayectoria en la investigación sobre organización empresarial; sin embargo, por lo general, se ha reducido únicamente a los trabajadores y ha excluido los niveles más altos de gobierno corporativo. En las modernas empresas, el valor aportado por el capital es cada vez más reducido, y el riesgo, en muchas ocasiones, es compartido por el conjunto de stakeholders de la empresa. Si la aportación de valor y la asunción de riesgos no son exclusivos de los shareholders, ipor qué los derechos de gobierno han de ser únicos para los shareholders? En este artículo se reflexiona sobre los fundamentos de la participación de los diferentes grupos de interés que componen la empresa. Se abordan los problemas vinculados a la instrumentalización de la Teoría de Stakeholders, y al agotamiento deductivo de la misma y se propone adoptar la perspectiva ontológica, así como un acercamiento práctico de carácter inductivo. La principal aportación del trabajo es ofrecer una guía metodológica en la que se resume la participación de los stakeholders en las organizaciones.

Palabras clave autores

Ética empresarial, gobernanza corporativa, teoría de los Stakeholders, psicología empresarial, participación, empresa ciudadana.

Palabras clave descriptores

Psicología organizacional, responsabilidad social empresarial, corporaciones.

\section{A B S T R A C T}

The participation in the company is a longstanding issue in research on business organization, but usually is explained around workers and not around the highest levels of corporate governance. In the modern businesses, the value contribution of capital is dwindling, and the risk, in many situations, is shared by all of the stakeholders of the company. If the added value and the assumed risk are not unique to the shareholders, why should be the government right unique for shareholder? In this paper we reflect on the foundations of the participation of different stakeholders that group the company. We debate the problems associated with the manipulation of Stakeholder Theory, and the depletion deduction of it; moreover, we propose to take the ontological perspective and a practical approach of inductive character. The main contribution of work is to provide a methodological guide which summarizes the participation in organizations of stakeholders. 
Key words authors

Business ethics, corporate governance, stakeholders theory, enterprise psychology, participation, citizenship enterprises. Key words plus Organizational psychology, corporate social responsibility, corporations.

\section{Introducción}

En la nueva economía, caracterizada por su dinamismo, emergen nuevos valores y formas de organización. De la importancia, casi exclusiva, del inmovilizado material en tiempos pasados se ha pasado a dar gran relevancia al valor de los intangibles que son difícilmente cuantificables pero no por ello menos importantes; de la estructura piramidal más o menos descentralizada a la de red; de la propiedad de los medios de producción al outsourcing. En general, los cambios en la concepción de las organizaciones y sus factores de valor han sido constantes en las últimas décadas. Sin embargo, en lo que a participación en la gobernanza corporativa se refiere, se sigue utilizando la teoría de los derechos de propiedad del capital, relegando la participación a un ámbito meramente operativo. Por ello, en el presente trabajo de investigación se pretende avanzar en la identificación de los fundamentos teóricos y procesos metodológicos que permitan avanzar en la creación de una cultura y unos valores donde la participación de los trabajadores y otros stakeholders de la organización en la gobernanza ${ }^{1}$ corporativa, sea una necesidad.

En esta línea de trabajo, se pretende analizar la pertinencia de la participación de los stakeholders en el marco de la gobernanza corporativa a partir de la revisión de las teorías sobre ese tema (Clarke, 2005), y en especial utilizando como justificación la

1 Los problemas para definir este concepto se han agravado desde el desarrollo de su proceso, condición o método. Existen al menos seis usos distintos de gobernanza (véase Rhode, 2005, p. 100), entre ellos el de gobernanza corporativa, sentido en el que se utilizará este concepto en el presente trabajo.
Teoría de los Stakeholders (Freeman, 1984), la Teoría Multifiduciaria (Boatright, 2002) y la Teoría de la Empresa Ciudadana (Neron \& Norman, 2008).

La nueva realidad de la empresa del siglo XXI debería llevar a un nuevo concepto de propiedad empresarial, donde esta no se vinculara de forma exclusiva a la aportación de capital, sino a la aportación de valor, en gran medida vinculada a la gestión de recursos y capacidades intangibles. Este planteamiento se encuentra muy cerca de la "revolución normativa" de Donaldson (2008), aunque el matiz relativo a la esencia de la empresa hace que redenominemos esta revolución como ontológica (referido a la esencia o núcleo del concepto de empresa), ya que no se trata fundamentalmente de lo que debería hacer la empresa (perspectiva normativa/ética), sino fundamentalmente de lo que es la empresa (perspectiva positiva, ontológica); de este ser sustancial se derivará lo que "debe hacer" la empresa (conclusiones normativas).

El objeto de este artículo consiste, por una parte, en revisar la gobernanza de la empresa a la luz de la perspectiva ontológica de la Teoría de Stakholders y, por otra parte, abordar el problema de la aplicabilidad de la participación de los stakeholders. Así, las principales aportaciones de este artículo son:

1. La fundamentación de la gobernanza en la empresa debe pasar de una perspectiva centrada en los shareholders a una perspectiva centrada en los stakeholders.

2. La metodología de participación de los stakeholders debe pasar de una comprensión del valor económico, a una concepción antropológica/ emocional, que integre todas las necesidades propias del ser humano (lúdicas, sociales, de sentido...).

\section{Revisión de la literatura}

\section{La participación en la gobernanza corporativa desde la teoría de los derechos de propiedad}

La participación de los trabajadores en la empresa es un tema ampliamente tratado en la investigación 
sobre recursos humanos y organización (Navarro, 2007), sin embargo, dicha participación siempre se ha fundamentado como una delegación del principal (accionista) a través del agente ( $\mathrm{CEO} /$ equipo directivo) y en beneficio de los resultados de la empresa, y por tanto de los accionistas.

En este sentido, se debe tener en cuenta que el principio fundamental de la teoría del derecho de propiedad puede definirse como que la propiedad de la empresa (entendida la propiedad en términos de capital de la organización) genera, al menos, dos derechos, el derecho a obtener el beneficio resultante de la actividad de la empresa y el derecho a decidir. El primer derecho surge de la identificación entre empresa y capital, es decir, el poseedor del capital es el dueño de la empresa y, por lo tanto, el único que tiene derecho sobre los resultados de esa empresa. El segundo derecho deviene del primero mediatizado por el factor riesgo, y puede enunciarse como un silogismo lógico tal que $\mathrm{A} \Lambda \mathrm{B} \otimes \mathrm{C}$ : puesto que los accionistas reciben un beneficio variable en función de los resultados de la empresa, y esos resultados se ven afectados por la gestión de la misma; los accionistas tienen derecho a tomar las decisiones en la empresa, lo que afecta a la gobernanza corporativa. El resto de los participantes en la empresa trabajadores, proveedores, clientes y otros, recibirán un beneficio o retribución pactada en función de la Teoría Contractual (Williamson, 2002).

Basándose en este doble planteamiento de la teoría de los derechos de propiedad y la teoría contractual, se ha generado la perspectiva denominada Teoría de los Shareholders y que de forma general se atribuye a Friedman (1962). Las decisiones en la empresa corresponden a los propietarios de la misma, en la que se presupone que los accionistas son los únicos que arriesgan en su constitución y, por lo tanto, corresponde a los mismos tomar las decisiones, ya que la gobernanza corporativa debe ir vinculada a la asunción de riesgos. No obstante, como señala la Teoría de Agencia, en las corporaciones, los propietarios no dirigen de forma directa, sino que lo hacen a través de los directivos a los que delegan los poderes propios de los propietarios (Jensen \& Meckling, 1976). Consecuentemente, los directivos, denominados Agentes, no hacen sino dirigir la empresa por delegación de los propietarios, a los que se denomina Principales; de lo que se deduce que la responsabilidad de los Agentes consiste fundamentalmente en optimizar los intereses de los Principales, aunque, a veces de forma ilícita, pueden anteponer sus propios intereses.

\section{La participación en la gobernanza corporativa desde la teoría de los stakeholders}

La Teoría de Stakeholders ha supuesto, sin duda, una revolución en cuanto a la forma de concebir la empresa y los derechos de propiedad del capital. En el ámbito de la teoría de empresa se han sucedido diversos planteamientos, que en gran medida han ido integrando los anteriores. En la siguiente figura (Figura 1) se representan gráficamente estos enfoques.

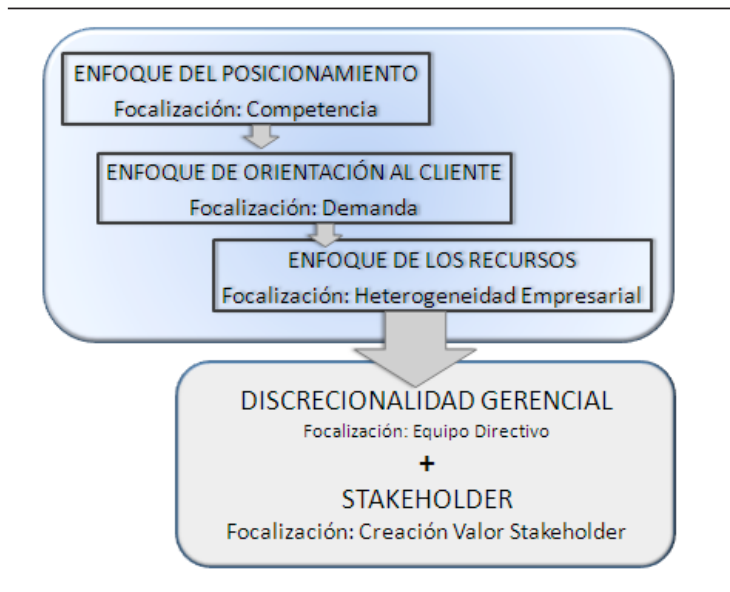

Figura 1. Evolución de la participación y la teoría de los stakeholders.

Fuente: elaboración propia.

Cada uno de estos planteamiento enfatiza algunas variables relevantes de la empresa, así la teoría de las cinco fuerzas de Porter (1985) destaca la posición competitiva, y por ende la importancia del sector. Casi en paralelo, el enfoque orientado al cliente (Kotler, 1988) enfatiza el papel que las necesidades de este deben tener en la estrategia de la empresa, y resaltan de forma significativa la importancia del stakeholder cliente, aunque la 
terminología utilizada sea otra. La Teoría de Recursos y Capacidades (Wernerfelt, 1984) desplaza la centralidad hacía los recursos idiosincrásicos de la empresa. Por su parte, la Teoría de la Discrecionalidad Gerencial (Hambrick \& Mason, 1984), aún en desarrollo, refocaliza la atención en la capacidad diferencial de la dirección de la empresa en relación con la organización de los recursos y capacidad, en el marco del contexto competitivo.

En este contexto, la Teoría de Stakeholders se presenta como una evolución lógica que toma en consideración todos los grupos de interés señalados en estas teorías, complementándolos con algunos ausentes, como los proveedores, el Gobierno, o la sociedad.

A partir de la constatación por parte de Friedman (1962) de la importancia que puede tener para los resultados de la empresa la preocupación por otros grupos de interés ajenos a los shareholders, adquiere carta de naturaleza el planteamiento instrumental de la Responsabilidad Social Corporativa. Pero no es hasta la aparición de la Teoría de los Stakeholders (Freeman, 1984) que la responsabilidad de la empresa con el conjunto de los grupos de interés, pasa a ser una realidad consustancial a la propia empresa, posibilitando tanto una perspectiva normativa como una perspectiva positiva de carácter ontológico.

La Teoría de los Stakeholders (Freeman, 1984), considera que las organizaciones están compuestas por un conjunto de participantes, a los que denomina grupos de interés (stakeholders), y entre los que habitualmente se incluyen: accionistas, trabajadores, financiadores, proveedores, clientes, administración, y sociedad en general. Desde esta perspectiva, la empresa aparece como el resultado de la interrelación de todos esos grupos a lo largo del tiempo (proceso diacrónico). Esta teoría se opone a los planteamientos anteriores, centrados exclusivamente en los accionistas, generándose una antítesis entre dos concepciones alternativas de la empresa, en lo que ha venido a denominarse el "debate Friedman-Freeman" (Freeman, Harrison $\&$ Wicks, 2008).

El planteamiento clásico, atribuido a Friedman se centra fundamentalmente en los grupos de presión, es decir aquellos que con sus actuaciones pueden perjudicar los resultados de la empresa, y proporciona un fundamento adecuado a la Responsabilidad Social Empresarial orientada desde el marketing. En este sentido, nos encontramos ante un planteamiento instrumental de la responsabilidad social, ya que la fundamentación de la misma no es de carácter ético u ontológico, sino utilitarista.

Antagónicamente, el planteamiento normativo de la responsabilidad social empresarial considera constituyentes y consustanciales a la empresa a la totalidad de los grupos de interés. Siendo la finalidad de la empresa y, por tanto, la de su equipo directivo satisfacer sus intereses de la mejor forma posible. Debido a que los intereses son divergentes, y en algunos casos contrapuestos, la dirección de la empresa deberá intentar satisfacerlos de forma equilibrada, sin priorizar un grupo respecto a otro de forma continuada a lo largo del tiempo (Freeman, Harrison, Wicks, Parmar \& de Colle, 2010). Mientras en la perspectiva instrumental el interés final de la organización se orienta en función de los shareholders, en la perspectiva normativa lo hace en función de todos los participantes en la empresa, lo cual tiene importantes repercusiones en los indicadores de valor utilizados, ya que posiblemente los beneficios -que pueden ser un buen indicador para los accionistas- posiblemente sean muy importantes para los vecinos o para los proveedores.

Fundamentación teórica de la participación de los stakeholders en la gobernanza corporativa

El tema de la participación, y especialmente de la fundamentación del derecho de participación, es un tema de actualidad en la Ética de la Empresa. Entre los trabajos disponibles, se destacarán tres propuestas. La primera corresponde a la de la Teoría de Stakeholders, con los recientes trabajos de Freeman et al. $(2008,2010)$ que conciben la empresa como un conjunto de relaciones con la finalidad de maximizar el valor para la totalidad de los stakeholders. En segundo lugar, la Teoría Multifiduciaria (Boatright, 2002; Goodpaster, 1991) que, basada en la teoría contractual y más específicamente en la de Agencia, 
otorga categoría de principal no solo a los accionistas, sino al conjunto de stakeholders, generando la obligación para el agente de crear valor para todos ellos. Por último, se relevaría la conceptualización de "Empresa Ciudadana" desarrollada por Neron y Norman (2008) que, al entender de los autores del presente trabajo, aporta la posibilidad de que las empresas incorporen la responsabilidad con los no stakeholders.

La Teoría Multifiduciaria, que es la que más se asemeja al planteamiento ontológico propuesto con anterioridad, nace como solución a la paradoja de Goodpaster (1991) que plantea, desde la Teoría de Agencia, el problema de que los directivos -los agentes-actúan únicamente como fiduciarios del principal -los accionistas-. Concluyendo que, puesto que su derecho se fundamenta únicamente en la cesión de derechos por parte de los propietarios, el agente no está legitimado para tomar decisiones que el propio principal no tomaría, o por las que este se sienta perjudicado. La paradoja presentada por Goodpaster reviste especial importancia por cuanto la Teoría de los Stakeholders postula la figura de la dirección como la encargada de equilibrar la satisfacción de los intereses del conjunto de stakeholders, resolviendo los conflictos de intereses que pudieran surgir entre ellos. Si el agente otorgara a grupos de stakeholders diferentes de los shareholders más de lo que estos estuvieran dispuestos a concederles, contravendrían el principio de fiduciaridad, dando lugar a un problema ético y legal.

La respuesta a esta paradoja genera la Teoría Multifiduciaria que se enmarca en la Teoría de Agencia. Sin embargo, reinterpreta la consideración de principal, ampliándola al conjunto de stakeholders, con lo que el agente estaría no solo al servicio de un solo grupo (los shareholders), sino de la totalidad de los grupos de interés. Su papel como articulador de un equilibrio en la satisfacción de intereses entre el conjunto de los stakeholders, quedaría así plenamente justificado.

No obstante, aunque la perspectiva multistakeholders soluciona de forma teórica el problema de participación de los mismos en los máximos niveles de gobernanza, se enfrenta a múltiples problemas, tanto teóricos como prácticos. Entre los teóricos destaca el problema de ingobernanza propuesto por Jensen (2002), el cual plantea que la Teoría Multifiduciaria resulta incompatible con el gobierno efectivo de la empresa, ya que al existir un conjunto de principales con intereses divergentes, e incluso contrapuestos, el agente adoptaría el rol de árbitro, con lo cual se pierde el posible control de los principales sobre el agente. Esta situación supondría que en la práctica el agente se convierte en principal, si no de derecho, sí de hecho. No obstante, este problema no puede ser considerado específico de un planteamiento multistakeholder, ya que en muchos casos el control del agente por parte del principal es ficticio. En la realidad, la actuación fiduciaria del agente respecto del principal se soporta en la responsabilidad ética del agente; consecuentemente, esa misma responsabilidad habría que suponérsela al agente ante un grupo heterogéneo de principales (Boatright, 2002). En opinión de los autores de este estudio el problema de control no se encuentra asociado tanto al conflicto entre intereses de los diferentes stakeholders, sino a la dispersión del poder entre los principales. Los conflictos de intereses se dan de igual forma entre los propios shareholders, y en los casos de no existencia de mayorías o de participaciones muy dispersas dicho poder recae de forma real en el CEO (chief executive officer) de la corporación. Es cierto que la Teoría Multifiduciaria introduce un nuevo factor cualitativo -al margen del cuantitativo de incremento y dispersión de los interesados-la inexistencia de estructuración legal a la participación del resto de stakeholders.

En cuanto a los problemas prácticos, resulta difícil resolver la ponderación y representación de los diferentes grupos de stakeholders en las máximas instancias de gobernanza de las organizaciones.

La propuesta de "Empresa Ciudadana" cuenta a su vez con varias aproximaciones: minimalista, equitativa y extendida, siendo esta última la que nos parece más interesante desde el punto de vista de la gobernanza y participación. Post y Berman (2001, p. 68) la definen acertadamente al considerar:

(...) la corporación ciudadana, como el proceso de identificación, análisis y respuesta a las responsabilidades sociales, políticas y económicas tal y como son definidas por la ley y las políticas públicas, las 
expectativas de los stakeholder y las actuaciones voluntarias derivadas de los valores corporativos y de las estrategias de negocio. La ciudadanía corporativa implica los resultados actuales (qué hace la compañía) y los procesos a través de los cuales se obtienen (cómo lo hace).

Por su parte, Neron y Norman (2008) realizan una aproximación muy interesante a través de la extensión de los derechos de ciudadanía, lo que crea un cauce de solución para uno de los principales problemas no resueltos por la Responsabilidad Social Corporativa y la Teoría de los Stakeholders: la responsabilidad ética de la empresa ante los no stakeholders.

Los tres planteamientos señalados se enfrentan a serios problemas: respecto a la Teoría de Stakeholders, ya se ha mencionado la Paradoja de Goodpaster (1991). Respecto a la perspectiva multifiduciaria se tiene el Problema de Gobernanza planteado por Jensen (2002), ya que múltiples principales con intereses diversos convierten al agente en principal "de hecho". A esta crítica Boatright contrapone el espejismo de control que se da en la teoría clásica de agencia. Por su parte el planteamiento de Nerón y Norman reúne críticas variadas, entre ellas por ejemplo la variabilidad del concepto de "buen ciudadano" según las culturas de los diferentes países (De George, 2008).

A pesar de sus indudables problemas, las tres teorías generan oportunidades muy interesantes en relación con la participación de todos o parte de los stakeholders en la gobernanza de la empresa, en el marco del modelo capitalista subyacente. Por su parte, la teoría multifiduciaria presenta una fundamentación sólida del derecho de participación, basado en la teoría contractual y en la de agencia, pilares de la actual teoría de empresa. Por otra parte, la "Gestión para los Stakeholders", que el propio Freeman (Freeman et al., 2008, p. 6) denomina como "capitalismo de los stakeholders", se sitúa como una vía intermedia entre el capitalismo y la economía social (Retolaza \& San-Jose, 2011), aportando una fundamentación y una propuesta metodológica que permiten una gestión basada en el conjunto de los stakeholders, y consecuentemente una participación de los mismos en todos los ámbitos de la empresa. Por último, el planteamiento de la "Empresa Ciudadana" justifica el interés legitimo por los no stakeholders, y por tanto su posible inclusión, directa o delegada, en los órganos de gobernanza de la empresa.

\section{Discusión y propuesta metodológica}

\section{Perspectiva antropológica del valor: satisfacción de intereses variados y personales}

Desde una perspectiva clásica, se identifica la creación de valor con generación de rentas; la monetarización de las rentas limita la producción de valor, convirtiendo al valor en un bien limitado. La apropiación de un bien limitado y deseable da lugar a un conflicto por la apropiación de las rentas que enfrenta a los agentes implicados: accionistas, trabajadores, proveedores, clientes, etc. El resultado de la distribución responderá a las relaciones de poder establecidas (Porter, 1985), esperándose como consecuencia de la resolución de dicho conflicto una situación de eficiencia óptima.

Siendo cierto que en la empresa existen conflictos reales por la apropiación de rentas, la teoría de stakeholders va más allá en el planteamiento a través de dos postulados: el primero hace referencia a que el valor producido no se equipara con la renta, sino con la satisfacción de intereses, y puede ser de naturaleza material o inmaterial. El segundo se refiere a la capacidad interactiva en la creación de valor entre los stakeholders. Esta interacción será positiva y se basa en la búsqueda del interés de la otra parte, generando un flujo de valor ilimitado, muy superior al valor monetario de la relación contractual entre partes.

El primer postulado, frente a la concepción fixista (fija) del valor que lo identifica con dinero, aborda el problema desde una óptima fenomenológica. El valor se define desde la importancia que le da aquella persona que lo recibe; normalmente en la recepción de bienes materiales existen estándares relacionales, por lo que es posible darles un valor monetario. Sin embargo, en el caso de la recepción 
de bienes inmateriales, no existe un estándar de transformación a valor monetario, por lo cual el valor es aquel que la persona receptora le atribuye, independientemente del valor que le puedan atribuir otras personas y del valor de producción.

Complementariamente, la generación de este tipo de valor entre varios stakeholders puede ser aditiva en lugar de sustractiva. Cuando se hace referencia a la satisfacción de interés de los stakeholders, la consideración fenomenológica del concepto de valor genera una nueva perspectiva que permite transcender el conflicto por la apropiación, ya que al tratarse de una generación ilimitada de valor puede haber una apropiación complementaria no conflictiva. En este sentido, la participación del conjunto de stakeholders en la gobernanza de la empresa puede posibilitar que este se oriente mejor a la consecución de "valor" para el conjunto de stakeholders. Como se ha señalado, dicho valor no tiene por qué ser monetario, ni tan siquiera económico, por lo que podría darse el caso de un incremento general en el valor percibido por cada stakeholder.

Asimismo, la generación de valor multistakeholder se ha estudiado recientemente en las Cajas de Ahorros Españolas, llegándose a la conclusión de que la participación de los stakeholders en la gobernanza no merma la generación de valor, sino que lo reorienta en función de los fines diferenciados de la entidad (San-Jose, Retolaza \& Torres, 2011).

\section{Propuesta metodológica para la participación de los stakeholders}

Podemos considerar que los cauces fundamentales de participación en la empresa de mayor a menor nivel son los siguientes: 1) la asamblea accionistas/ socios: órgano de máxima nivel de la organización. Salvo en el caso de algunas entidades de economía social, en las entidades mercantiles la participación está basada en el derecho de propiedad del capital y limitada a los accionistas. Actualmente, resulta legalmente difícil el poder dar participación no accionarial a los stakeholders. Participaciones accionariales minoritarias les pueden dar relevancia (voz) en la asamblea, si el agente lo facilita, aun cuando no se tenga poder real (voto). La reestructuración de la legislación para permitir la existencia de sociedades mercantiles en las que los derechos de los socios no estén vinculados con la propiedad del capital, es una asignatura pendiente en las legislaciones de la mayoría de los países; la "agrupación de interés económico" puede ser una fórmula de referencia.

Por su parte, el Consejo Administración, máximo órgano ejecutivo de la empresa, está vinculado al concepto de propiedad ya que actúa como delegación de la misma pero tiene posibilidades de reorientarse desde la teoría Multifiduciaria, ya que al igual que ha incorporado consejeros independientes, podría incorporar consejeros que representaran los intereses de diferentes stakeholders. Las conductas oportunistas de dichos consejeros podrían constituirse en uno de los principales problemas.

La Junta Directiva y los equipos de dirección tienen posibilidades de incorporar representantes de otros stakeholders; sin embargo, se da la paradoja de que una vez incorporados pasarían al grupo de stakeholders de dirección, y su grupo de adscripción inicial -consumidores, vecino u otrosquedaría relegado a un nivel secundario. Cualquier persona perteneciente a un grupo de stakeholders a la que se incorpore como trabajador estable en la empresa, pasará a ser primariamente del grupo de stakeholders de trabajadores/directivos, quedando relegado su anterior grupo de interés y no pudiendo, por tanto, ser representante del mismo.

Los Equipos de Mejora, dado su carácter temporal, pueden facilitar la incorporación de representantes de los stakeholders, lo cuales pueden actuar como facilitadores en la incorporación de los intereses de un mayor número de stakeholders en la estrategia y actuaciones de la empresa.

Por último, pero no menos importante, puede darse una participación individual sistematizada a través de cauces formales o informales de recogida y gestión de la información. Aunque para que realmente este tipo de participación pudiera asociarse con la gobernanza debería tener adscrita alguna cuota de poder en la decisión; las modernas tecnologías hacen cada día más fácil la realización de consultas vinculantes a los interesados, lo cual podría suponer una forma difusa de incorporarlos en la gobernanza de las entidades. 
Como síntesis, se puede señalar que la incorporación de los stakeholders en la asamblea de accionistas/socios sería deseable, pero imposible sin cambios legislativos previos, así mismo sería imposible su incorporación a puestos de dirección por la "paradoja de cambio de rol" que se ha señalado. Los cauces de participación más interesantes se encuentran en el Consejo de Administración, en los grupos temporales de mejora, y a través de la participación en procesos abiertos de decisión, que podrían orientarse en los procesos de innovación abierta que tan buenos resultados están generando (Chesbrough, 2003).

En gran medida, la responsabilidad fiduciaria del agente en relación al principal no descansa en la necesidad de control, sino que en muchas ocasiones recae en la responsabilidad del agente, como señala la Stewardship Theory (Donaldson \& Harris, 1991). En este sentido, no se quiere finalizar el artículo sin hacer una síntesis de los diferentes mecanismos propuestos tanto desde la Teoría de Stakeholders como desde el liderazgo ético en relación con el desarrollo de una gestión orientada al conjunto de stakeholders, independientemente de que sea imposible su incorporación en los órganos de gobernanza.

Desde la perspectiva de los stakeholders, el trabajo más interesante a la fecha es el de Freeman et al. (2008), quienes proponen una serie de principios y herramientas que permiten potenciar la gestión hacía los stakeholders, siendo los principales: 1) definición y compromiso con los principios, 2) elaboración de un mapa de stakeholders, 3) especificación de los valores y estrategias de la empresa en relación con los stakeholders, 4) métodos concretos de creación de valor y 5) liderazgo ético capaz de canalizar la realidad actual de la empresa hacía los planteamientos normativos propuestos.

Freeman et al. (2008) describen los siguientes diez principios para la gestión de stakeholders. 1) Los intereses de los diferentes stakeholders son simultáneos en el tiempo. 2) Se deben encontrar soluciones que satisfagan a los distintos stakeholders de forma simultánea. 3) No se pueden satisfacer los intereses de una parte en perjuicio de otra(s) de forma continuada en el tiempo. 4) Se debe actuar con el propósito de satisfacer las expectativas de todos los stakeholders. 5) Se debe permitir a los stakeholders participar en el gobierno corporativo. 6) Es necesaria una continua interacción y diálogo con todos los stakeholders. 7) Los stakeholders son personas reales con nombre y caras. 8) Es necesario tener una perspectiva de marketing. 9) Hay que prestar atención tanto a los stakeholders primarios como secundarios. 10) Se deberán analizar y rediseñar continuamente los procesos, para dar un mejor servicio a los stakeholders.

Todas ellas se centran en los stakeholders y en la inclusión de sus intereses en la empresa; sin embargo, el quinto principio hace referencia explícita a la participación de los stakeholders en los órganos de gobierno, respecto al cual cabe plantearse cómo se soporta o se sustenta legalmente, aspecto aún pendiente de abordar en la literatura científica.

\section{La clave de la gobernanza multistakeholder: el liderazgo ético}

Como señala Jensen (2002), la ampliación de la responsabilidad de la empresa al conjunto de stakeholders otorga un papel aún más relevante al agente, es decir, a los directivos de la organización, incrementando simultáneamente la dificultad del principal o principales para poder realizar la función de control, ampliando el conocido "problema de agencia" (Jensen \& Meckling, 1976). En este contexto, adquiere especial relevancia la disposición del agente a asumir los objetivos del conjunto de principales, siendo capaz de anteponerlos a sus legítimas aspiraciones. Se está hablando del liderazgo ético, ya que difícilmente puede haber otro tipo de liderazgo que en una situación de no control anteponga los intereses de los stakeholders al beneficio propio.

A falta de un desarrollo específico del tema del liderazgo ético, se apuntan dos propuestas interesantes, las $7 \mathrm{C}$ del liderazgo ético de Moreno (2004): 1) Carácter, voluntad y hábitos, 2) Coraje de saber decir no, 3) Credibilidad: coherencia, consecuencia y confianza, 4) Comunicar: claro y conciso, 5) Conocimiento: competencia y capacidad, 6) Compromiso: trabajo y organización y 7) Com- 
TABLA 1

Principios para la participación de los stakeholders en la empresa

\begin{tabular}{ll}
\hline \multicolumn{1}{c}{ Principio } & \multicolumn{1}{c}{ Descripción } \\
\hline del Líder & $\begin{array}{l}\text { El líder es un miembro de la propia organización y de un grupo de stakeholders. Sus actuaciones } \\
\text { deben ser en beneficio del conjunto de stakeholders. }\end{array}$ \\
de los Integrantes & $\begin{array}{l}\text { El líder no ve a los integrantes como partes separadas, sino como un todo, e intenta desarrollar } \\
\text { una cultura conjunta de equipo1. }\end{array}$ \\
de los Resultados & Debe conectar los objetivos de la organización con los intereses individuales de los integrantes. \\
del Proceso: & Debe trabajar de forma abierta, integrando la perspectiva, valores y opiniones de los integrantes. \\
del Contexto & Debe basarse en principios éticos, pero debe ser flexible en las decisiones complejas. \\
Ético & Debe argumentar sus actuaciones y propuestas en términos éticos. \\
\hline
\end{tabular}

Fuente: elaboración propia a partir de Freeman et al. (2008).

prensión, y los principios propuestos por Freeman et al. (2008), recogidos en la Tabla 1.

\section{Conclusiones}

La participación de los trabajadores en la empresa es un tema ampliamente estudiado en la literatura; sin embargo, la ampliación de la participación a otros stakeholders como los clientes, proveedores, ciudadanía o Gobierno es un tema novedoso de gran actualidad. La Teoría de los Stakeholders, especialmente desde la perspectiva multifiduciaria u ontológica, permite fundamentar no solo la participación de los trabajadores, sino la del resto de los grupos de interés implicados en la empresa. $\mathrm{Si}$ bien es cierto que presenta algunos problemas y paradojas, también es cierto que las soluciones propuestas la sitúan como una de la teoría más potente en la actualidad en relación con la gobernanza corporativa.

La Teoría de Stakeholders se encuentra ampliamente aceptada, tanto por investigadores como por los gestores de las empresas más importantes del mundo (Agle \& Agle, 2007). Sin embargo, el deslizamiento hacía planteamientos instrumentales y la incapacidad de generar modelos prácticos de aplicabilidad, hacen que se pueda encontrar con que la teoría resulta inoperante en la realidad. Así pues, los mayores problemas no son de índole teórica sino práctica, y se centran en los mecanismos que pueden hacer posible la participación: legales, sociales y de gestión. Las principales propuestas se han centrado en el papel del liderazgo ético que debe desempeñar el agente; sin embargo, siendo esto relevante, no puede ocultarse la necesidad de modificar la estructura de gobernanza de las empresas en las que se permitan el acceso legal de los stakeholders a los máximos niveles de decisión.

En este contexto, el artículo realiza dos aportaciones fundamentales: la primera se refiere al interés de enfocar la Teoría de Stakeholders desde una perspectiva ontológica, lo que puede posibilitar que se convierta, incluso, en un nuevo paradigma para entender la empresa y las relaciones de esta con las personas y la sociedad. La segunda aportación hace referencia a una ampliación en el concepto de valor, evitando la reducción monetarista del mismo, la cual justifica más fácilmente el conflicto por la apropiación de rentas frente a la generación dialógica de valor que sería característica de la Teoría de Stakeholders.

En su conjunto, estas aportaciones pueden permitir el desarrollo de un nuevo modelo de aplicabilidad de la Teoría de Stakeholders, por ello la principal línea de investigación que se sigue es la de desarrollar un modelo funcional de aplicabilidad que se pueda usar de forma sistemática para incorporar a los sistemas de gestión habituales: plan estratégico, cuadro de mando integral y plan anual de gestión, satisfacción de los intereses de los stakeholders como objetivo de la empresa. En esta línea, un reto actual consistiría en definir y contrastar modelos de gobernanza participativos que se soporten legalmente o, en su defecto, ser capaces de identificar y proponer las modificaciones legislativas necesarias. 


\section{Referencias}

Agle, B. \& Agle, L. (2007). The stated objectives of the Fortune 500: Examining the philosophical approaches that drive America's largest firms (Working Paper). University of Pittsburgh, Pittsburgh, Pennsylvania, United States.

Boatright, J. R. (2002). Contractors as takeholders: Reconciling Stakeholders Theory with the Nexus-of Contracts Firm. Journal of Banking and Finances, 26(9), 1837-1852.

Chesbrough, H. W. (2003). Open innovation: The new imperative for creating and profiting from technology. Boston, MA: Harvard Business School Press.

Clarke, T. (2005). Theories of corporate governance. London: Routledge.

De George, R. T. (2008). Reflections on Citizenship, Inc. Business Ethics Quarterly, 18(1), 43-50.

Donaldson, T. (2008). Two stories. En B. R. Agle, T. Donaldson, R. E. Freeman, M. C. Jensen, R. K. Mitchell \& D. Wood (Contributors). Dialogue: Toward Superior Stakeholders Theory. Business Ethics Quarterly, 18(2), 172-176.

Donaldson, T. \& Harris, J. (1991). Stewardship Theory or Agency Theory: CEO Governance and Shareholder Returns. Australian Journal of Management, 16(1), 49-64.

Freeman, R. E. (1984). Strategic management: A stakeholder approach. Boston: Pitman Publishing.

Freeman, R. E., Harrison, J. S. \& Wicks, A. (2008). Managing for stakeholders: Survival, reputation, and success. New Haven, CT: Yale University Press.

Freeman, R. E., Harrison, J. S., Wicks, A., Parmar, B. L. \& de Colle, S. (2010). Stakeholder Theory: The state of the art. Cambridge, UK: Cambridge University Press.

Friedman, M. (1962). Capitalism and freedom. Chicago: University of Chicago Press.

Goodpaster, K. E. (1991). Business ethic and stakeholder analysis. Business Ethics Quarterly, 1(1), 53-73.

Gutiérrez, E. (2006). Cultura, organizaciones e intervención. Universitas Psychologica, 6(1), 115-129.

Hambrick, D. C. \& Mason, P. A. (1984). Upper echelons: The organization as a reflection of its top managers. Academy of Management Review, 9(2), 193-206.
Jensen, M. C. (2002). Value maximization, stakeholder theory, and the corporate objective function. Business Ethics Quarterly, 12(3), 235-256.

Jensen, M. C. \& Meckling, W. H. (1976). Theory of the firm: Managerial behavior, agency costs and ownership structure. Journal of Financial Economics, 3(4), 305-360.

Kotler, P. (1988). Marketing management: Analysis, planning, implementation, and control. Englewood Cliffs, NJ: Prentice Hall.

Moreno, C. M. (2004). Las 7 C del liderazgo ético. Capital Humano, 183, 84-89.

Navarro, J. A. (2007). Críticas y apoyos hacia la responsabilidad social de la empresa: una aproximación etnográfica desde la vivencia del trabajador y una propuesta metodológica cualitativa. Universitas Psychologica, 6(1), 59-68.

Neron, P. Y. \& Norman, W. (2008). Citizenship, Inc.: Do we really want businesses to be good corporate citizens? Business Ethics Quarterly, 18(1), 1-26.

Porter, M. (1985). Competitive advantage: Creating and sustaining superior performance. New York: Free Press.

Post, J. E. \& Berman, S. L. (2001). Global corporate citizenship in a Dot.com world: The role of organisational identity. En J. Andriof \& M. Mcintosh (Eds.), Perspectives on corporate citizenship (pp. 6683). Shefield, Eng: Greenleaf Publishing.

Retolaza, J. L. \& San-Jose, L. (2011). Social economy and stakeholder theory, an integrative framework for socialization of the capitalism. CIRIEC-España, Revista de Economía, 73, 193-213.

Rhode, R. A. W. (2005). La nueva gobernanza: gobernar sin gobierno. En A. Cerrillo (Coord.), La gobernanza hoy: 10 textos de referencia (pp. 99-123). Madrid: INAP.

San-Jose, L., Retolaza, J. L. \& Torres, J. (2011). The efficiency of banking institutions: Spanish saving banks versus Spanish commercial banks from a multi-stakeholder perspective. International Research Journal of Applied Finance, 2(7), 925-935.

Wernerfelt, B. (1984). A resource-based view of the firm. Strategic Management Journal, 5, 171-180.

Williamson, O. E. (2002). The theory of the firm as governance structure: From choice to contract. Journal of Economic Perspectives, 16(3), 171-195. 